\title{
Using Distances to Classify Recordings of Young and Elderly Subjects
}

\author{
Stavroula Vlachothanasi, George Manis \\ University of Ioannina, Dept. of Computer Science and Engineering, Ioannina, Greece
}

\begin{abstract}
To the large number of diverse methods for the computation of heart rate variability and the common classification algorithms we add a new one, based on the distances between heart rate signals. Given two groups of subjects and a test subject, which we want to categorize into one of the two groups, we compute the average distance of the test subject from each group and then classify the subject based on these two distances.

In this algorithm, we do not use distances between groups offeatures, but distances between the signals themselves. The surprising result is that the signals are not categorized simply based on the minimum average distance from each group. We noticed that distances between signals of elderly subjects were smaller that those between young subjects. Distances between young and elderly subjects were somewhere in the middle. Three distance metrics are used: the Euclidean distance, the Manhattan distance and dynamic time warping.
\end{abstract}

\section{Introduction}

A large number of methods estimating the variability of the heart rate has been proposed. Many of them are used in the clinical practice giving significant information to the physicians for the condition of the heart and possible hidden irregularities. In [1] guidelines for the standards of measurement, the physiological interpretation, and the clinical use of Heart Rate Variability (HRV) are presented. The review paper [2] comes to give attention to newer methods, or methods that were given not much attention in [1].

Some of the proposed methods are mainly used in the research, seem promising, present remarkable results, but are not widely used in clinical practice. This is not because these methods are less important, but due to the slow adapting times that physicians normally (and is necessary to) present.

One may wonder why there are so many methods already proposed and why every day new ones appear. The answer is simple and must be sought to the complexity of the heart as an organ and as a structural unit. This com- plexity has not been modeled in a satisfactory degree and we are still far away from it. What we have achieved by now is to observe its electrical activity and interpret the recorded information. The underlying phenomena that influence the heart rhythm have also not deeply investigated, even though medicine and technology have made huge steps during the last decades. Since we do not know the accurate mechanism that produce the heat rhythm, we cannot decide which method is the most appropriate one. We are not even sure if such a method exists.

Therefore, papers with new methods appear often and been accepted for publication. Each method attempts to depict the phenomenon from a different point of view, emphasizing on the information that can describe better. Lets stay in a coarse grain level and see what kind of information is attempted to be captured from each family of common methods.

Time domain methods analyze the signals describing the variability in the time domain. No matter if they exam the differences between successive beats, or the differences from the mean, or something else, what they actually compute is the variability of the signal in a given time interval.

Frequency domain methods describe the power in underlying rhythms. Even though there is a correspondence between methods of time and frequency domains, the expression of the variability is done with different means and the information extracted is not always the same. This is the reason why both these families of methods are widely used in the clinical practice, but none of them has been accepted as the only standard.

The wide use and acceptance of time and frequency domain methods has led to the examination of time-domain methods. Even though these methods are used, at least until now, by the researchers only, the results show that breaking the signal into scales and then applying time domain methods in each scale can sometimes give more clear information.

Non linear methods see the problem from a completely different point of view. They consider the heart as a complex system and try to measure this complexity. Looking deeper in this family we will see a large number of different ways to do that.

In conclusion, the complexity of the phenomenon we 
study and the relatively low knowledge of its underlying mechanisms have led us to adopt a large set of diverse methods that aim to describe the variability of the heart rhythm from a different point a view. A completely different approach is proposed in this paper, adding one more perspective to the existing ones.

In this paper we compare signals and we categorize them based on their distances from other signals. Given two groups of subjects expected to present different variabilities and one heart rate signal for which we want classify into one of the two groups, we compute the mean distance of the signal from all signals belonging in the first group and all signals belonging in the second group. Comparing these two average values we can classify the examined signal. Distances between signals of elderly subjects were smaller that those between young subjects. The classification criterion is not the minimum distance between the two groups, as one could normally expect. Distances between young and elderly subjects were somewhere in the middle. In our experiments we used three distance metrics: i) Euclidean distance (ED) ii) Manhattan or City Block distance (MD) and iii) Dynamic Time Warping (DTW).

The rest of the paper is structured as follows. Section 2 presents the three metrics used to compute the distances between two signals. Section 3 is divided into three subsections, describing the data we used, the experiments we did and our results. The last section concludes this work.

\section{Distance Metrics}

The distance between two signals can be expressed with many different metrics. When the signals are of the same size, the Euclidean distance can be employed. Suppose we have the signals $x_{i}$ and $y_{i}$, where $1 \leq i \leq N$. The Euclidean distance is given by:

$$
D_{\text {eucl }}=\sqrt{\sum_{i=1}^{N}\left(x_{i}-y_{i}\right)^{2}}
$$

In a similar way, the Manhattan distance is defined. It is also called City Block distance:

$$
D_{\text {manh }}=\sqrt{\sum_{i=1}^{N}\left|x_{i}-y_{i}\right|}
$$

Another way to express the distance between two signal is by using the Dynamic Time Warping (DTW) metric. We will use the symbol $D_{d t w}$ in accordance with the other two metrics. $D_{d t w}$ can be used even when the two signals have not the same size. The method compares the two signals and corresponds points between them, so that the total sum of the distances between these points is the minimum. Figure 1 shows the details of the implementation.

Since the computation of DTW is of complexity $O\left(n^{2}\right)$, the computation of the distance between a given signal and

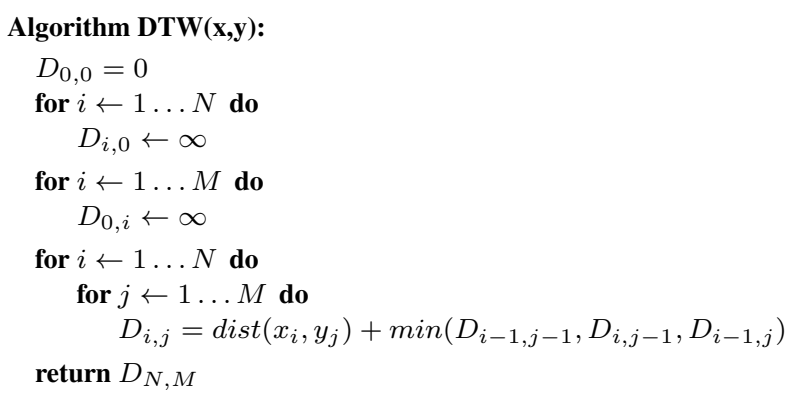

Figure 1. The computation of the distance of two signals $x, y$ usind $d t w$. The distance is given by the element $D_{N, M}$

all signals in a given group of signals can be very expensive. Especially, when the number of the signals in the group is large or/and the size of each signal is also large. In order to speedup the computation, we used a fast version of DTW.

DTW has been used in analyzing data from different scientific fields. In [3] DTW is used to find patterns in time series, in [4] for voice recognition, and in [5] for word image matching. We should mention the study [6] which uses DTW to compare electroencephalograms (EEGs). In this study the method PROCESS is introduced, in which EEGs are categorized in one of two groups, based on the DTW distance and k-NN classification algorithm. In this method, a vector with all distances of a signal from a given set of signals is fed to k-NN algorithm. The system is trained with a subset of the signals and tested with the rest of them. The results show that this method can classify EEGs with a remarkable accuracy.

\section{Experiments}

\subsection{Data}

For our experiments we used the Fantasia dataset [7]. This is a publicly available dataset which can be freely loaded from Physionet [8][9]. It consists of 40 recordings, 20 of which have been acquired from young subjects of age between 21 and 34 years old, and 20 from elderly subjects all between 68 and 85 years. All subjects were healthy. Each subgroup includes equal numbers of men and women. All subjects remained in a resting position and in sinus rhythm during recording. They were watching the movie Fantasia (Disney, 1940) to help maintain wakefulness. The continuous ECG signals were 120 minutes long and digitized at $250 \mathrm{~Hz}$. Each heartbeat was annotated using an automated detection algorithm, and each beat annotation was verified by visual inspection. We downloaded and used the $R R$ interval signal (heart rate signal). 
Table 1. Accuracy of Categorization

\begin{tabular}{c|ccc}
\hline Size & $\begin{array}{c}\text { Euclidean } \\
\text { Accuracy }(\%)\end{array}$ & $\begin{array}{c}\text { DTW } \\
\text { Accuracy }(\%)\end{array}$ & $\begin{array}{c}\text { Manhattan } \\
\text { Accuracy }(\%)\end{array}$ \\
\hline 64 & $97.90 \pm 2.31$ & $94.00 \pm 3.84$ & $98.45 \pm 1.57$ \\
128 & $98.90 \pm 1.67$ & $96.55 \pm 2.87$ & $99.00 \pm 1.58$ \\
256 & $98.90 \pm 1.51$ & $97.45 \pm 2.26$ & $99.70 \pm 0.81$ \\
512 & $98.40 \pm 1.48$ & $98.40 \pm 1.30$ & $99.80 \pm 0.68$ \\
1024 & $99.35 \pm 1.10$ & $99.20 \pm 1.17$ & $99.90 \pm 0.49$ \\
2048 & $99.65 \pm 0.87$ & $99.05 \pm 1.21$ & $99.90 \pm 0.49$ \\
4096 & $99.25 \pm 1.15$ & $99.85 \pm 0.59$ & 100.00 \\
\hline
\end{tabular}

\subsection{Methods}

We performed three different groups of experiments, each for one of the three metrics: $D_{e u c l}, D_{m a n h}$ and $D_{d t w}$. Each experiment is described by the following steps:

- step 1: in a preprocessing step, we normalize the original signals by dividing them with their mean value

- step 2: in the main part, we use leave-one-out cross validation to estimate the accuracy of the method

- step 2.1: we randomly select a subpart of each signal of size $N$

- step 2.2: for each of these subparts we compute the average distance between the time series belonging in the same category and the average distance between time series belonging in the different category

- step 2.3: we test if the classification failed or not

- step 3: finally, we compute the accuracy based on the leave-one-out cross validation

We performed each experiment 50 times and reported the mean accuracy. The testing hypothsis was that distances between signals of elderly subjects were smaller that those between young subjects. Distances between young and elderly subjects were somewhere in the middle.

Code was developed in Python and SL [10].

\subsection{Results}

In Table 1 the experimental results are shown. We can notice that the classification accuracy is remarkably high for all three metrics. The best results are presented by the Manhattan distance, which reaches to an $100 \%$ accuracy for $N=4096$. The accuracy increases as $N$ increases as expected. Since all compared signals were of the same size, DTW could not exploit its ability to compare signals of different size. We kept the size of compared signals equal in order to have comparable results and to reduce the numbers of the parameters of the problem.

\section{Conclusions}

Each family of the common heart rate variability analysis methods estimates the variability from a different point of view. Statistical methods estimate variability in time domain, frequency methods estimate the power in specific frequencies, time-frequency methods are somewhere in the middle, when non-linear methods estimate the complexity of the underlying system. The methodology proposed here is different: it compares distances between the signals and categorizes them based on these distances. As metrics we used the Euclidean, the Manhattan Distance and Dynamic Time Warping.

We used the Fantasia dataset available from Physionet. It consists of recordings of 20 young and 20 elderly subjects. With the RR time series we performed the following experiment. We first normalized the signals by dividing them with their means. Then, we randomly selected a subpart of each, with length $N$. We computed the average distance of each signal from all signals belonging in the same group and from all signals belonging in the other group. We noticed that distances between signals of elderly subjects were smaller that those between young subjects. Distances between young and elderly subjects were somewhere in the middle. We used leave-one-out cross validation to estimate the accuracy of the method. We performed the experiment 50 times and report here the average accuracy.

The results showed high accuracy, almost always higher than $95 \%$, even for very small values of $N$. For $N=4096$ the accuracy reaches to $100 \%$ for the Manhattan distance.

\section{References}

[1] Task Force of the European Society of Cardiology the North American Society of Pacing Electrophysiology. Heart rate variability: Standards of measurement, physiological interpretation, and clinical use. Circulation 1996;93(5):10431065.

[2] Sassi R, Cerutti S, Lombardi F, Malik M, Huikuri H, Peng $\mathrm{C}$, Schmidt G, Yamamoto Y. Advances in heart rate variability signal analysis: Joint position statement by the eCardiology ESC Working Group and the European Heart Rhythm Association co-endorsed by the Asia Pacific Heart Rhythm Society. Europace 2015;17(9):1341-53.

[3] Berndt DJ, Clifford J. Using dynamic time warping to find patterns in time series. In Proceedings of the 3rd International Conference on Knowledge Discovery and Data Mining, AAAIWS'94. AAAI Press, 1994; 359-370.

[4] Muda L, Begam M, Elamvazuthi I. Voice recognition algorithms using Mel Frequency Cepstral Coefficient (MFCC) and Dynamic Time Warping (DTW) techniques. Journal of Computing March 2010;2(3).

[5] Rath TM, Manmatha R. Word image matching using dynamic time warping. In 2003 IEEE Computer Society Con- 
ference on Computer Vision and Pattern Recognition, 2003. Proceedings., volume 2. June 2003; II-521-II-527.

[6] Buza K, Koller J, Marussy K. Process: projection-based classification of electroencephalograph signals. In International Conference on Artificial Intelligence and Soft Computing. 2015; 91-100.

[7] Iyengar N, Peng CK, Morin R, Goldberger AL, Lipsitz LA. Age-related alterations in the fractal scaling of cardiac interbeat interval dynamics. The American Journal of Physiology October 1996;271(4 Pt 2).

[8] Goldberger AL, Amaral LAN, Glass L, Hausdorff JM, Ivanov PC, Mark RG, Mietus JE, Moody GB, Peng CK, Stanley HE. PhysioBank, PhysioToolkit, and PhysioNet: Components of a new research resource for complex physiologic signals. Circulation 2000 (June 13);101(23):e215- e220.

[9] PhysioNet. URL https: / / physionet.org/.

[10] Saougkos D, Manis G. A parallelizing compiler for the microgrid: Exploiting concurrency from software continuity. In the AppleCore Project Workshop: Making Multi-cores Mainstream. 2012; .

Address for correspondence:

George Manis

University of Ioannina, Dept. of Computer Science and Engineering, P.O. Box 1186, Ioannina, 45110, Greece.

manis@cs.uoi.gr 